\title{
Identifying, Collecting, and Presenting Hacker Community Data: Forums, IRC, Carding Shops, and DNMs
}

\author{
Po-Yi Du, Ning Zhang, Mohammedreza Ebrahimi, Sagar Samtani, Ben Lazarine, Nolan Arnold, Rachael Dunn, \\ Sandeep Suntwal, Guadalupe Angeles, Robert Schweitzer, Hsinchun Chen \\ Department of Management Information Systems, The University of Arizona \\ Tucson, AZ 85721, USA \\ \{pydu, zhangning, ebrahimi, sagars, benlazarine, nolanarnold, rajacobi, sandeepsuntwal, angeles, rschweitzer\}@email.arizona.edu, \\ hchen@eller.arizona.edu
}

\begin{abstract}
Cyber-attacks cost the global economy over $\$ 450$ billion annually. To combat this issue, researchers and practitioners put enormous efforts into developing Cyber Threat Intelligence, or the process of identifying emerging threats and key hackers. However, the reliance on internal network data to has resulted in inherently reactive intelligence. CTI experts have urged the importance of proactively studying the large, everevolving online hacker community. Despite their CTI value, collecting data from hacker community platforms is a non-trivial task. In this paper, we summarize our efforts in systematically identifying and automatically collecting a large-scale of hacker forums, carding shops, Internet-Relay-Chat, and DarkNet Marketplaces. We also present our efforts to provide this data to the larger CTI community via the AZSecure Hacker Assets Portal (www.azsecure-hap.com). With our methodology, we collected 102 platforms for a total of 43,902,913 records. To the best of our knowledge, this compilation of hacker community data is the largest such collection in academia, and can enable a numerous novel and valuable proactive CTI research inquiries.
\end{abstract}

Keywords - Cybersecurity; Hacker community data collection; Hacker forums; Internet-Relay-Chat; DarkNet Marketplaces; Carding Shops; cyber threat intelligence

\section{INTRODUCTION}

With computer and information technology becoming more ubiquitous, cybersecurity has become a grand societal challenge. Today, malicious hackers commit numerous largescale, advanced attacks on industry and government organizations. These cyber-attacks cost the global economy over $\$ 450$ billion annually [1]. Cyber Threat Intelligence (CTI), or the process of identifying emerging threats and key threat actors (i.e., hackers) to enable effective cybersecurity decisions, has emerged as a viable approach to mitigate this concern.

Fundamentally a data-driven process, CTI has traditionally focused on collecting data from internal network devices databases, IDS/IPS, routers, workstations, and others. Collected data is analyzed using malware analysis, forensics, event correlation, and other well-established methods. Despite the prevalence of these approaches, CTI experts from major cybersecurity firms, such as the SANS Institute, note that the reliance data from past events (i.e., internal network data) results in inherently reactive intelligence [2]. As a result, cyberattacks remain on an unfortunate uptick.
To combat this issue, CTI experts have urged the importance of developing proactive CTI by directly investigating hackers within the online hacker community [2][3]. The international online hacker community attracts and motivates millions of hackers from the US, Russia, and China, to share or sell hacking tools, knowledge, and other illegal products and services [4]. Today, four major hacker community platforms exist: hacker forums, Internet-Relay-Chat (IRC), carding shops, and DarkNet Marketplaces (DNMs). Exploits found on these platforms have executed well-publicized attacks such as the BlackPOS malware for the Target breach or the Mirai botnet for the internet-scale Denial-of-Service (DoS) attack in 2016.

Despite each platform's significant CTI value, collecting their data is a non-trivial task. Hacker community platforms carefully conceal themselves and employ numerous anticrawling measures that prevent automated, large-scale data collection. These barriers force many researchers to manual collection efforts. Studies attempting automated collection are limited to one platform type. In this paper, we summarize our work in identifying and automatically collecting a large-scale of hacker forum, carding shop, IRC, and DNM data. We also present our efforts to provide this data to the larger CTI community via the AZSecure Hacker Assets Portal. To the best of our knowledge, this collection of hacker community data is the largest in academia. Consequently, it can enable a multitude of novel and valuable proactive CTI research inquiries.

The remainder of this paper is organized as follows. First, we review each platform, discuss their CTI value, and note existing data collection strategies. Section III details our platform identification and collection methodology. Section IV summarizes our collected data and highlights promising CTI research directions. Section V illustrates key functions of the Hacker Asset Portal. Section VI concludes this work.

\section{HACKER COMMUNITY PlatForm REVIEW}

\section{A. Hacker Community Platforms Overview}

To the best of our knowledge, four hacker community platforms exist: (1) forums, (2) IRC, (3) DNMs, and (4) carding shops. Taken together, these platforms have hundreds of millions of records made by millions of hackers across the 
globe. Table I describes each platform and their CTI value.

TABLE I. HACKER COMMUNITY PLATFORM SUMMARY

\begin{tabular}{|c|l|l|}
\hline Platform & \multicolumn{1}{|c|}{ Description } & \multicolumn{1}{c|}{ CTI Value } \\
\hline $\begin{array}{c}\text { Hacker } \\
\text { Forums }\end{array}$ & $\begin{array}{l}\text { Message board } \\
\text { allowing members to } \\
\text { post messages that are } \\
\text { archived }\end{array}$ & $\begin{array}{l}\text { Key threat actor identification; } \\
\text { sharing of hacking tools; indication } \\
\text { of access to other hacker } \\
\text { communities }\end{array}$ \\
\hline IRC & $\begin{array}{l}\text { Plain-text, instant } \\
\text { messaging, } \\
\text { communication that is } \\
\text { not archived }\end{array}$ & $\begin{array}{l}\text { Sharing of hacking knowledge and } \\
\text { potential target; indication of } \\
\text { access to other hacker communities }\end{array}$ \\
\hline DNMs & $\begin{array}{l}\text { Markets on Tor that } \\
\text { sell illicit goods via } \\
\text { cryptocurrency }\end{array}$ & $\begin{array}{l}\text { Early indicator for breached } \\
\text { companies; in-depth understanding } \\
\text { of underground economy }\end{array}$ \\
\hline $\begin{array}{c}\text { Carding } \\
\text { Shops }\end{array}$ & $\begin{array}{l}\text { Shops selling stolen } \\
\text { credit/debit cards and } \\
\text { sensitive data }\end{array}$ & $\begin{array}{l}\text { Monitoring trafficking of internet } \\
\text { fraud industry; precaution of } \\
\text { breaches before happen }\end{array}$ \\
\hline
\end{tabular}

The four hacker community platforms create an ecosystem of hacker activities. Hackers use forums and/or IRC to freely discuss and share Tools, Techniques, and Processes (TTP) and advertise hacking services. Hackers freely download these tools or navigate to DNMs to purchase exploits. These tools help hackers conduct cyber-attacks to attain sensitive data such as credit/debit cards, Social Security Numbers (SSN), and logins to sell on DNMs and/or carding shops for financial gain. Each platform is further detailed in the following sub-sections.

\section{1) Hacker Forums}

Hacker forums are the most common and largest platforms for hackers to share hacking resources [4]. Hackers use these message boards to post messages within threads of conversations related to hacking tools, techniques, and malicious source code. Among the four major platforms, forums are the only one allowing hackers to post malicious exploits for others to freely download [5]. Figure 1 illustrates an example of a hacker sharing ransomware.

The open sharing of hacking assets enables individuals with limited hacking skills to become capable of conducting cyberattacks [4]. This characteristic, combined with the rich metadata (e.g., post content, post date) make forums a viable data source for monitoring the TTP of hackers, identifying key actors, and discovering emerging threats.

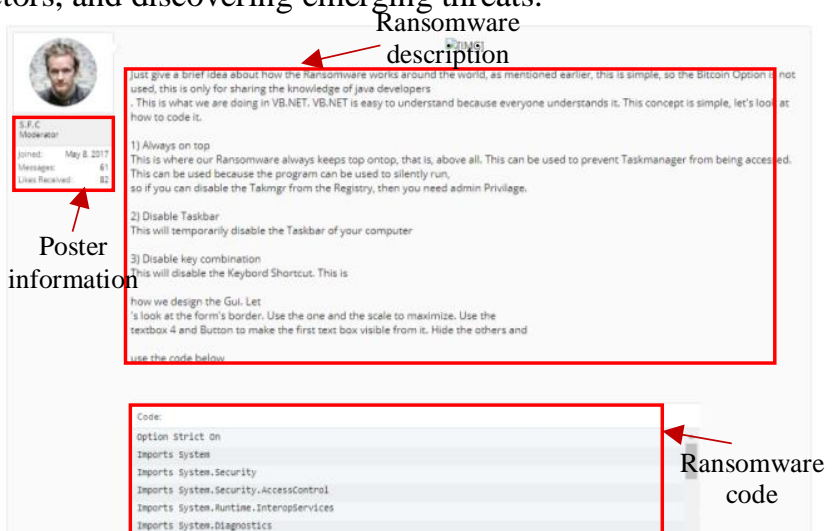

Figure 1. An example of a hacker forum member sharing ransomware code

\section{2) Internet-Relay-Chat (IRC)}

Built on a separate protocol, an IRC server can hold multiple channels, containing conversations about pre-defined topics [4]. Although not originally intended for hackers, IRC channels have become a popular medium for hacktivist groups to share hacking knowledge. Figures 2 and 3 illustrate two examples of user behaviors on hacker IRC. Figure 2 depicts hackers sharing links to forums with illegal contents. Figure 3 illustrates an IRC user demanding hacking service with a provided target IP.

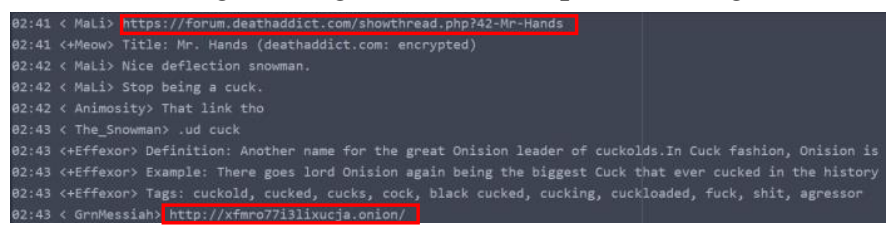

Figure 2. An example of hackers sharing links containing illegal contents $11: 59$ < Gustav> hack this ip 172.98 .79 .37
$11: 59$ < Gustav> ddos it
$11: 59$ < Gustav> it's my school
$12: 00$ < Gustav> I love you Figure 3. An example of an IRC user demanding hacker service

Unlike forums, IRC conversations are not archived and must be collected in real-time. Additionally, IRC messages are broadcast to all channel participants. If a user loses server connection, he/she cannot retrieve the conversation for that time period [6]. This allows hackers to share hacking knowledge and targets more freely. As a result, collecting IRC data can help understand hacker behaviors, targets, and emerging threats.

\section{3) DarkNet Marketplaces (DNMs)}

DNMs operate as Tor hidden services, and are one of the major platforms on which users make illegal transactions with cryptocurrency [7]. Products such as drugs, hacking tools, weapons, and stolen personal documents can easily be found on DNM. Access to DNMs are often indicated by other hacker communities such as hacker forums and IRC channels [4].

Two categories of DNM data exist: product and seller. Each product listing contains a name, description, price, delivery destinations, and product category. Seller information includes seller history, ID, rating and trust level, PGP keys, etc. Figure 4 illustrates an example of a product listing page on DNM, where a scam page of PayPal to conduct phishing attacks is sold.

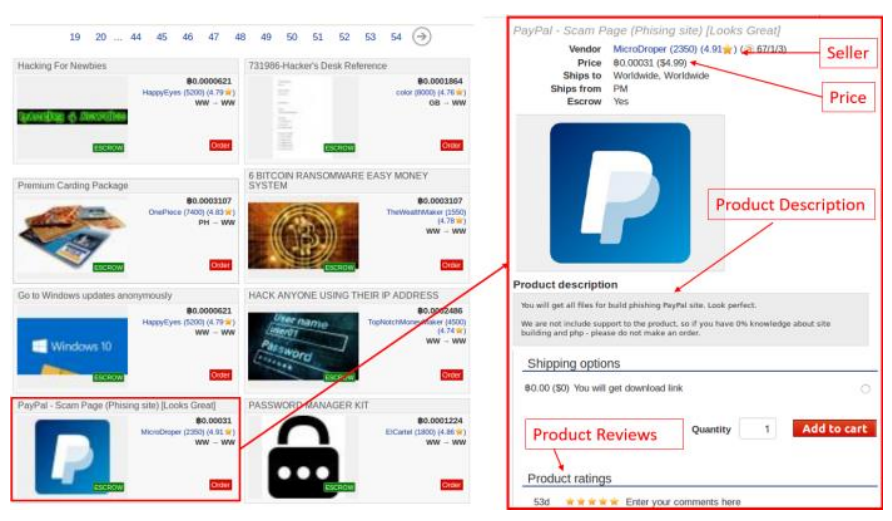

Figure 4. An example of a product listing page on DNM

Data and information stolen from breached companies are 
often sold on DNM. Thus, DNMs can serve as an early indicator for breached organizations. Also, past research indicate the thriving of DNM has raised concern in public health and law enforcement, for holding an abundant amount of drug listing [8][9]. Researchers observed that DNM users often share information about reliable seller and quality goods among DNM [9][10]. Moreover, DNM has been leveraged to explore the product distribution network [11]. While Tor's untraceable nature makes linking DNM users to their true identity is a nontrivial task, studying their behavior can provide an in-depth understanding about the underground economy.

\section{4) Carding Shops}

Carding shops facilitate many underground economy activities by providing high quality carding services [4]. A large amount of stolen card data from attained from data breaches are traded and diffused through carding shops [12]. Similar to DNMs, access to carding shops are often indicated in other hacker community platforms. Since carding data are duplicable and recyclable, the rapid dissemination of card information can inflict significant financial losses on cardholders.

Carding shop data can be divided into payment card data, identity data, and credential data [13]. Payment card data is the major product type in carding shop, and it can be further categorized into "Dumps" and "Fullz" based on the amount of information carried by the product. Dumps refer to the raw information retrieved from the magnetic stripe of the card. Fullz includes the full information of the victim, including name, address. Both Dumps and Fullz contain three sections: card information, source, and price. Beyond these two, SSNs and logins are also commonly found on carding shops.

Carding shops have unique CTI value as they provide a comprehensive view of carding fraud. Despite its importance, little academic literature exists about them [4]. Past researchers have analyzed relationships between each attribute and price by comparison to identify that card data is packaged, with a label, to periodically release on carding shop [12]. Despite this useful discovery, significant potential remains for carding shop data to be used as a source of identifying exploited individuals and financial institutions.

\section{B. Hacker Community Data Collection: Existing Approaches and Challenges}

Unlike traditional social media sites, researchers face numerous issues when collecting hacker community platforms. Web-based platforms (i.e., forums, shops, DNMs) employ anticrawling measures such as drive-by malware, session timers, user-agent checks, CAPTCHA, and others. IRC data must be collected in real-time. These challenges have limited many researchers to manual collection efforts, or to studying old datasets (e.g., dumped SilkRoad, archived forums). While still valuable, such procedures result in incomplete and/or dated CTI insights. Studies employing automated collection procedures are often limited to one platform type (e.g., forums). However, each hacker community platform is interconnected with others. Thus, the focus on collecting one platform prevents comprehensive CTI development. These issues motivate the development of large-scale, automated crawling approaches.

\section{Collection Methodology}

We developed a systematic approach to gather a comprehensive collection of hacker community data. The process has three phases, platform identification, enhanced automated collection, and content parsing. We summarize each in the following sub-sections.

\section{A. Hacker Community Platform Identification}

The first stage in any data collection task is identifying the appropriate data sources. We use three approaches to identify hacker platforms: suggestions from cybersecurity experts, surface web and Tor search engines, and snowball identification. Using all three ensure a comprehensive, high-quality coverage. Irrespective of approach, we only collected platforms containing significant amounts of cybersecurity content. We deliberately avoided platforms specializing in weapons or pornography, as such content has minimal CTI value.

In the first strategy, our team consulted with the National Cyber-Forensics Training Alliance (NCFTA), a major nonprofit organization focusing on the CTI sharing across private, public, and academic sectors, and Policing in Cyberspace (POLCYB), an internationally recognized law enforcement entity. Both suggested platforms providing valuable cybersecurity data and also recommended keywords as input for surface web and Tor-based (e.g., Grams, Deep Dot Web) search engines to identify additional platforms. Since hackers often information on traditional social media platforms (e.g., Twitter, Facebook, YouTube), these keywords were also inputted into these sites to identify additional platforms. Figure 5 depicts a YouTube video of Anonymous recruiting for their IRC channel, \#OpTestet.

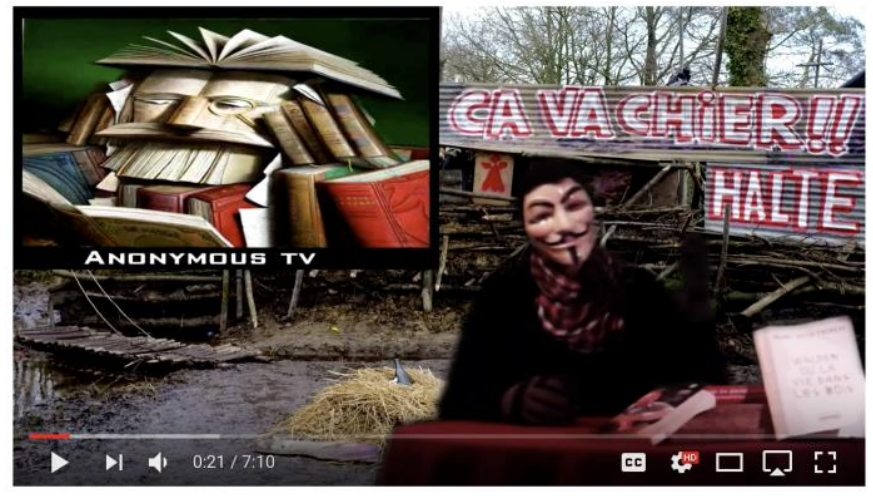

[ENG VERSION] Anonymous $>$ \#OpTestet ZAD Partout / Barrage de Sivens TESTET

Figure 5. An example of a recruiting video of Anonymous on YouTube

The platforms identified from the first two approaches were used as "seeds" for our final strategy: snowball identification. Hackers within these platforms often post links to other platforms. We followed these links and identified if they contained valuable cybersecurity content. The newly identified platforms were used as new seeds to identify additional platforms. 


\section{B. Enhanced Automated Collection Procedures}

Collection processes for hacker community data vary. Past web-based platforms (i.e., forums, DNMs, carding shops) research usually conducted undirected web crawlers to collect the raw data in the HTML format. To address the anti-crawling challenges detailed in our review, we upgraded our crawler to directly collect web pages and contents. Moreover, we utilized packet capture technology to overcome the challenge that some of websites restrict user to skip specific pages. By flexibly switching types of HTTP request, we significantly reduced the time cost on crawling web-based hacker platform data. For IRC data, we employed two "bots," similar to fake users, inside each channel, and used these bots to log in at their own routines, to avoid automatic disconnections.

\section{Content Parsing}

After data collection, the collected raw data requires further parsing to enable subsequent analytics. For forums, DNMs, carding shops, and IRC, parsing entails recognizing text patterns containing relevant attributes (e.g., post date, product description). We developed several custom parser programs and leveraged techniques such as Regular Expression (RegEx) to retrieve information from those platforms, and stored them into a relational database.

\section{Data Collection OVER VIEW}

In our hacker community data collection, we successfully collected 51 hacker forums, 13 IRC channels, 12 DNMs, and 11 carding shops. Table II summarizes our collection.

TABLE II. HACKER COMMUNITY DATA COLLECTION SUMMARY

\begin{tabular}{|c|c|c|c|}
\hline Platform & \# of Platform & \# of Records & Languages \\
\hline Forums & 51 forums & $3,643,216$ posts & $\begin{array}{c}\text { English/ Russian/ } \\
\text { Arabic }\end{array}$ \\
\hline IRC & 13 channels & $\begin{array}{c}2,791,120 \text { lines } \\
\text { of conversation }\end{array}$ & English \\
\hline DNM & 12 markets & 291,616 listings & $\begin{array}{c}\text { English/ Russian/ } \\
\text { French }\end{array}$ \\
\hline Carding Shops & 26 shops & $\begin{array}{c}8,674,087 \\
\text { listings }\end{array}$ & English \\
\hline
\end{tabular}

\section{A. Hacker Forums}

We focused on collecting 51 hacking oriented forums containing 32,266,852 posts in 2,961,363 threads. 25,939,871 are in English, 5,975,821 are in Russian, and 2,624,658 are in Arabic. Generally, we observed high frequency of hacking/security tools, for instance, online shopping site receipt generators for phishing purpose. Some forums specialize in other services such as breached data, mobile malware, cryptocurrencies, login dumps, and code for AI bots. The multilingual feature of our collection can facilitate cybersecurity research in cross-countries comparison.

Due to the popularity and ease of access, hacker forum data has a unique advantage that might not be seen in other hacker community platforms. The prolific nature of forum as well as their dynamic and time-sensitive property enables the researcher to identify trends of cyber threats easier and sometimes earlier than other platforms. Hence, a promising research direction would be developing time-sensitive methods to analyze the trends of cyber threat landscape through constant monitoring of the forum data. Another direction would be cross-referencing the forum data with DNMs in order to have holistic trend analysis. Moreover, due to the interactive structure of these platforms, they are capable of revealing the interaction network of cyber criminals.

\section{B. $I R C$}

We collected 2,791,120 lines of conversation from 13 IRC cybersecurity specific IRC channels between 9/2016 and 1/2018. The data collection is summarized in Table III. For space consideration, we only listed the top six channels.

TABLE III. IRC DATA COLLECTION SUMMARY

\begin{tabular}{|c|c|c|}
\hline Channel & \# of lines & Description \\
\hline \#anonops & $1,696,024$ & $\begin{array}{c}\text { General discussion of hacking-related } \\
\text { topics }\end{array}$ \\
\hline \#ed & 574,024 & Discussion about current topics \\
\hline \#hackers & 174,328 & $\begin{array}{c}\text { General discussion of tips and tricks for } \\
\text { Anonymous hackers }\end{array}$ \\
\hline \#Evilzone & 163,402 & Casual discussions on cyber security \\
\hline \#ddos & 23,172 & $\begin{array}{c}\text { Posts about current ddos tools } \\
\text { recommended by Anonymous hackers } \\
\text { \#tutorials } \\
\text { through a separate interactive IRC } \\
\text { channel }\end{array}$ \\
\hline $\begin{array}{c}\text { Total (of all } \\
\text { channels) }\end{array}$ & $\mathbf{2 , 7 9 1 , 1 2 0}$ & \\
\hline
\end{tabular}

The most popular IRC channel "anonops" is the main channel of the famous hacktivist group, Anonymous. Anonymous also runs channels such as "ddos," which focuses on Distributed Denial-of-Service (DDoS), and "hacker," in which users share and ask for hacking tips. IRC users also demand/provide hacking services with target information to each other. While past studies have explored the IRC participant duration [14], the CTI value of IRC data is still undiscovered. The links, URLs, and named entities exchanged in IRC chatrooms can be used in a snowball sampling manner to expand cyber threat resources. After identifying resources, a promising research direction would be discovering conversation topics via topic modeling approaches (e.g., Latent Dirichlet Allocation). Techniques such as Named Entity Recognition (NER) and relationship extraction can identify the targets of hackers and hacktivists in IRC.

\section{DNM}

We collected 12 DNMs between September, 2016 and January, 2018. Table IV summarizes our DNM data collection.

TABLE IV. DNM DATA COLLECTION SUMMARY

\begin{tabular}{|c|c|c|c|}
\hline DNM & $\begin{array}{c}\text { \# of } \\
\text { listing }\end{array}$ & $\begin{array}{c}\text { \# of security } \\
\text { listing }\end{array}$ & Language \\
\hline Oday & 28,330 & $28,330+$ & English \\
\hline Alphabay & 25,118 & N/A & English \\
\hline Apple Market & 2,012 & N/A & English \\
\hline Dream Market & 120,962 & $1,916+$ & English \\
\hline French Deep Web & 1,536 & $134+$ & French \\
\hline
\end{tabular}




\begin{tabular}{|c|c|c|c|}
\hline Hansa & 14,149 & N/A & English \\
\hline Minerva & 166 & N/A & English \\
\hline Russian Silk Road & 488 & N/A & Russian \\
\hline SilkRoad3 & 1,798 & $70+$ & English \\
\hline TradeRoute & 35,504 & $547+$ & English \\
\hline TOCHKA & 1,958 & $300+$ & Russian/English \\
\hline Valhalla & 17,576 & $695+$ & English \\
\hline Total: & $\mathbf{2 4 9 , 5 9 7}$ & $\mathbf{3 1 , 9 9 2 +}$ & $\begin{array}{c}\text { English/Russian/ } \\
\text { French/Dutch }\end{array}$ \\
\hline
\end{tabular}

All DNMs, except for Oday, contain illicit product not limited to cybersecurity. Among these DNMs, $60 \%$ of the products are drug related. Cybersecurity-related products account for around $20 \%$, while the remainder are weapon and stolen personal document. Oday data, on the other hand, contains only exploits. Such listings display information about the exploit category, description, the platform affected the exploit, and severity level. Most of the listings were originally priced. Once a patch or fix for the exploit comes out, the listing becomes free. In our collection, only 37 out of 28,330 exploits are priced, and the rest are free. Figure 6 is an example of Oday exploit listing.

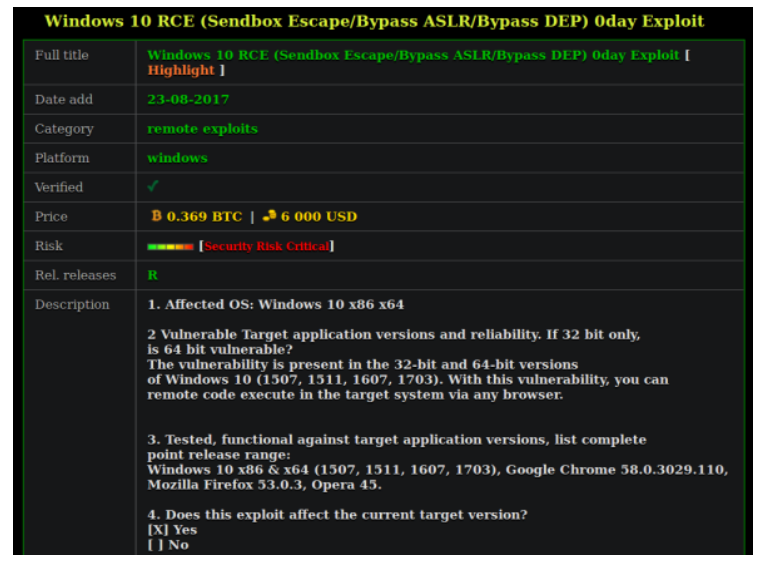

Figure 6. An example of a 0day exploit listing

Since that DNMs are relatively new compared to other platforms, there seems to be more untapped research opportunities in this area. The research done by Nunes et al. (2016) is one of the few studies that specifically target this area in which 11,992 DNM products were collected and semisupervised classification were employed to find the hackingrelated products. Based on the collected DNM data we observe that there is a rise in the number of non-English marketplaces. More specifically, multi-lingual identification of threats across marketplaces with different languages such as Russian and French would add a global insight of threats.

Cross-platform studies can be expanded beyond analyzing DNMs with different languages. We found some overlap between cyber threats being advertised in DNMs and forums. Studying the supply chain aspects of the threats between these two platforms is another possible promising research area that can provide insights about the dissemination patterns and flow of the cyber-security related products in the span of time.

\section{Carding Shops}

From 5/2014 to $1 / 2018$, we collected 26 carding shops data with $8,674,078$ listing. The majority are credit card dumps (6,596,093 listings), followed by Fullz and SSNs at 1,999,251 and 78,734 respectively. Table V summarizes our collection. For space considerations, we only listed the top nine shops and BuySSN, the only shop with personal information.

TABLE V. CARDING SHOPS DATA COLLECTION SUMMARY

\begin{tabular}{|c|c|c|c|c|}
\hline Name & $\begin{array}{c}\text { \# of CVV and } \\
\text { Fullz }\end{array}$ & \# of Dumps & $\begin{array}{c}\text { \# of } \\
\text { SSN }\end{array}$ & Total \\
\hline Jokers Stash & 566,600 & 446,642 & 0 & $1,013,242$ \\
\hline DUMPS MANIA & 42,311 & 777,634 & 0 & 819,945 \\
\hline Buybest & 9,008 & 790,325 & 0 & 799,333 \\
\hline United Dumps & 63,575 & 734,495 & 0 & 798,070 \\
\hline $\begin{array}{c}\text { THE MONEY } \\
\text { TEAM }\end{array}$ & 20,462 & 750,642 & 0 & 771,104 \\
\hline EBIN CC & 13,786 & 752,441 & 0 & 766,227 \\
\hline Golden Shop & 19,732 & 727,888 & 0 & 747,620 \\
\hline Getcc Shop & 609,770 & 0 & 0 & 609,770 \\
\hline $\begin{array}{c}\text { BuySSN } \\
\text { Total (of all } \\
\text { shops) }\end{array}$ & $\mathbf{1 , 9 9 9 , 2 5 1}$ & $\mathbf{6 , 5 9 6 , 0 9 3}$ & $\mathbf{7 8 , 7 3 4}$ & $\mathbf{8 , 6 7 4 , 0 7 8}$ \\
\hline
\end{tabular}

Nine shops focus on selling payment card information. Six of them, such as Getcc Shop and Diamond Dumps, contain only Fullz data. Generally, price for payment card data greatly varies, and similar card information is frequently found across different shops. BuySSN (78,734 records), specializes in selling SSNs. Such information is significantly more capable of causing serious personal losses than do debit/credit cards.

One promising CTI research direction is identifying customers whose data have been breached. That is, having both names and zip code of each stolen card, would help identify the customer with an acceptable precision which would be useful for credit companies and law enforcement agencies. Given that both DNMs and carding shops often share credit card information, cross-referencing the stolen data on carding shop platforms with DNMs provides new insights about the dissemination patterns of breached data in the entire ecosystem.

\section{InTEGRATION INTO THE HACKER ASSETS PORTAL}

A key aspect of the CTI process is presenting relevant collected data and selected analysis for user consumption. This often comes in the form of an interactive system. Adhering to key requirement for effective CTI, we integrate collected data into one of our projects, the Hacker Assets Portal (www.azsecure-hap.com). Funded by the National Science Foundation (NSF), the Portal was designed to provide selected hacker community content for students in Scholarship-forService (SFS) programs to enhance their education such that they can develop proactive CTI measures when they assume government positions [15]. Initially hosting only forum data, the Portal has now includes all relevant records from DNMs and carding shops. Users can search, sort, and browse through content based on each platform's metadata. For example, users 
interested in identifying whether their credit/debit card was stolen can search their name. Should it appear, they can pinpoint which shop it is sold in, the card price, and others.

Beyond these core functionalities, we developed visualizations for users to interactively explore the data. Carefully constructed based on internal feedback provided by SFS students and external suggestions from NCFTA and POLCYB, each visualization can be filtered and sorted based on a user's needs. Figures 7 provides one panel of our carding shop dashboard, which provides an overview of active and expired cards as well as a geo-spatial breakdown of their locations and associated frequencies.

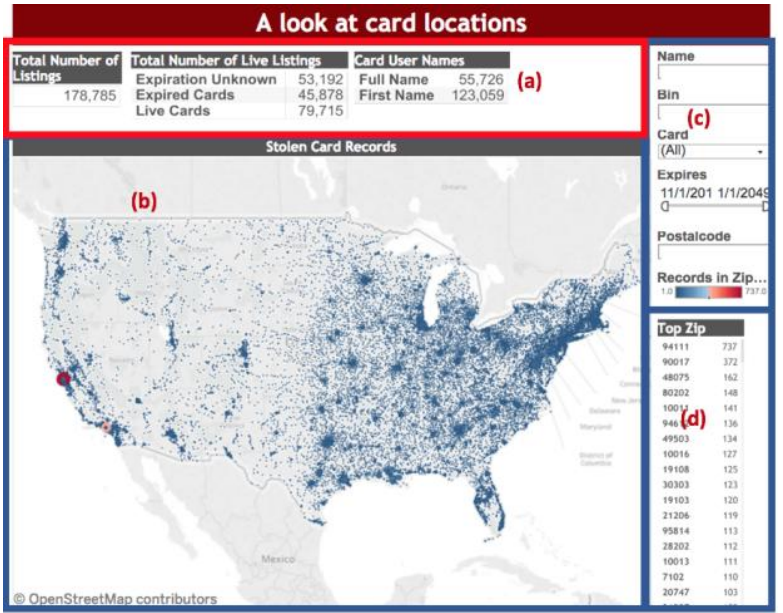

Figure 7. (a) scorecard of active and expired cards, (b) locations, (3) search, sort, and filter functions, and (d) frequency of cards based on zip code

Users can get more in-depth intelligence on selected platforms with finer grained visualizations. Figure 8 provides an example of a carding shop dashboard allowing users to dynamically compare shops based on frequency of cards, average prices, and banks of stolen cards.

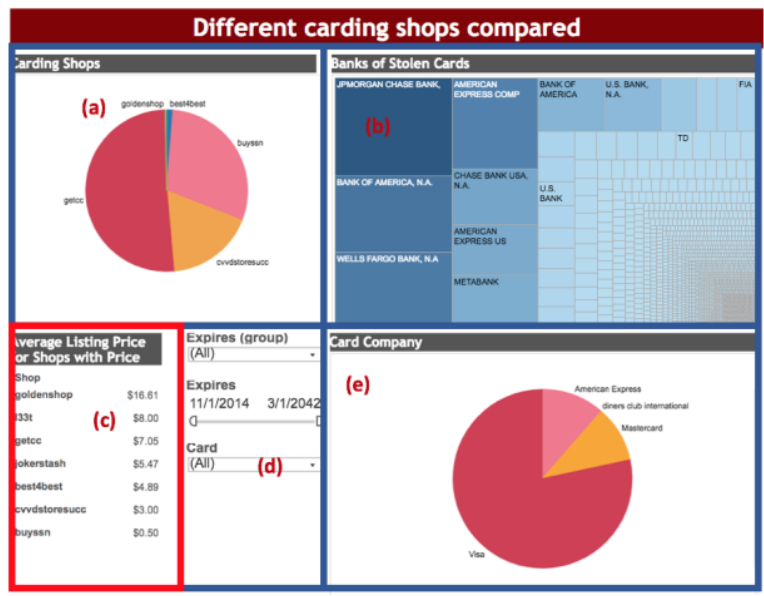

Figure 8. (a) frequency of cards per shop, (b) banks of stolen cards, (c) average card prices, (d) filter capabilities, and (e) card issuers with most stolen cards

\section{CONCLUSion AND Future Directions}

Cybersecurity has become a societal concern. CTI can provide a valuable process to pinpoint and defend against cyber-attacks. Today, the online hacker community has emerged as a valuable data source to generate proactive CTI. In this paper, we summarized our efforts to systematically identify and collect four major hacker community platforms: hacker forums, IRC, carding shops, and DarkNet Marketplaces. Through our methodology, we collected 102 platforms a total of 43,902,913 records. This large-scale collection enables numerous novel CTI research possibilities, including cross platform analysis, multi-lingual analysis, and others. Each direction can significantly improve our understanding of the hacker community, create proactive CTI, and most importantly, help develop a more secure society.

\section{ACKNOWLEDGEMENTS}

This work was supported in part by the National Science Foundation (NSF) DUE-1303362 (SFS), SES-1314631 (SaTC), ACI-1443019 (DIBBs), and 1719477 (EAGER).

\section{REFERENCES}

[1] Graham, L. 2017. "Cybercrime costs the global economy $\$ 450$ billion,” CEO. 7 Feb. 2017. https://www.cnbc.com/2017/02/07/cybercrime-costs-the-globaleconomy-450-billion-ceo.html

[2] Bromiley, M. (2016). "Threat Intelligence: What it is, and how to use it effectively." SANS Institute. https://www.sans.org/readingroom/whitepapers/analyst/threat-intelligence-is-effectively-37282

[3] Shackleford, D. (2016). "Security Analytics Survey" SANS Institute. https://www.sans.org/reading-room/whitepapers/analyst/2016security-analytics-survey-37467

[4] Benjamin, V., Li, W., Holt, T., \& Chen, H. (2015, May). Exploring threats and vulnerabilities in hacker web: Forums, IRC and carding shops. In Intelligence and Security Informatics (ISI), 2015 IEEE International Conference on (pp. 85-90). IEEE.

[5] Samtani, S., Chinn, R., Chen, H., \& Nunamaker Jr, J. F. (2017). Exploring Emerging Hacker Assets and Key Hackers for Proactive Cyber Threat Intelligence. Journal of Management Information Systems, 34(4), 1023-1053.

[6] Benjamin, V., Zhang, B., Nunamaker Jr, J. F., \& Chen, H. (2016). Examining hacker participation length in cybercriminal Internet-relaychat communities. Journal of Management Information Systems, 33(2), 482-510.

[7] Christin, N. (2013, May). Traveling the Silk Road: A measurement analysis of a large anonymous online marketplace. In Proceedings of the 22nd international conference on World Wide Web (pp. 213-224). ACM.

[8] Corazza, O., Schifano, F., Simonato, P., Fergus, S., Assi, S., Stair, J., ... \& Blaszko, U. (2012). Phenomenon of new drugs on the Internet: the case of ketamine derivative methoxetamine. Human Psychopharmacology: Clinical and Experimental, 27(2), 145-149.

[9] Van Hout, M. C., \& Bingham, T. (2014). Responsible vendors, intelligent consumers: Silk Road, the online revolution in drug trading. International Journal of Drug Policy, 25(2), 183-189.

[10] Davey, Z., Schifano, F., Corazza, O., Deluca, P., \& Psychonaut Web Mapping Group. (2012). e-Psychonauts: conducting research in online drug forum communities. Journal of Mental Health, 21(4), 386-394.

[11] Broséus, J., Rhumorbarbe, D., Mireault, C., Ouellette, V., Crispino, F., \& Décary-Hétu, D. (2016). Studying illicit drug trafficking on Darknet markets: structure and organisation from a Canadian perspective. Forensic science international, 264, 7-14.

[12] Bulakh and M. Gupta, "Characterizing credit card black markets on the web," in 2015, . DOI: 10.1145/2740908.2742128.

[13] Li, W., Yin, J., Chen, H. (2016). Identifying high quality carding services in underground economy using nonparametric supervised topic model. International Conference on Information Systems. Dublin, Republic of Ireland

[14] Benjamin, V., \& Chen, H. (2014, September). Time-to-event modeling for predicting hacker IRC community participant trajectory. In Intelligence and Security Informatics Conference (JISIC), 2014 IEEE Joint (pp. 25-32). IEEE.

[15] Samtani, S., Chinn, K., Larson, C., and Chen, H. "AZSecure Hacker Assets Portal: Cyber threat intelligence and Malware Analysis" In Intelligence and Security Informatics (ISI), 2016 IEEE International Conference on (pp. 19-24). IEEE. 